\title{
Metastasis of myxoid liposarcoma to fat-bearing areas: A case report of unusual metastatic sites and a hypothesis
}

\author{
SHUCHEN LIN $^{1 *}$, ZHIHUA GAN $^{1 *}, \mathrm{KUN} \mathrm{HAN}^{1}$, YANG YAO $^{2}$ and DALIU MIN ${ }^{1}$ \\ ${ }^{1}$ Department of Oncology, Shanghai Sixth People's Hospital East Campus, Shanghai Jiao Tong University, Shanghai 201306; \\ ${ }^{2}$ Department of Oncology, The Sixth People's Hospital, Shanghai Jiao Tong University, Shanghai 200233, P.R. China
}

Received October 14, 2014; Accepted July 7, 2015

DOI: 10.3892/ol.2015.3585

\begin{abstract}
Sarcoma is a rare disease that always metastasizes to the lungs and bones. Myxoid liposarcoma (MLPS), in comparison with other sarcomas, has a distinct biological characteristic. Recent studies have suggested that MLPS expresses high levels of adipophilin and chemokine (C-X-C motif) receptor 4 (CXCR4), which are correlated with adipogenesis and metastasis. In addition, the Ewing sarcoma breakpoint region 1-DNA damage-inducible transcript 3 (EWSR1-DDIT3) fusion transcript, recently identified in MLPS, was shown to selectively repress the osteoblastic transcription in multipotent mesenchymal cells. The present study reported a rare case of MLPS with metastasis in fat-bearing areas, including the bones, epidural region, orbits and abdominal cavity, while the lungs were not involved. Bone metastasis was diagnosed by magnetic resonance imaging and proven by histology. However, bone scanning lacked sufficient sensitivity to detect the bone metastasis. Based on these findings, we hypothesized that molecular adipophilin and molecular CXCR4 may contribute to the fat-bearing area metastasis pattern. Furthermore, the EWSR1-DDIT3 fusion transcript may repress the osteoblastic activity in the bone metastases, ultimately resulting in a low detection rate by bone scans.
\end{abstract}

\section{Introduction}

Liposarcoma (LPS) contains three subtypes: i) Well-differentiated LPS and dedifferentiated LPS; ii) myxoid LPS (MLPS); and iii) pleomorphic LPS. MLPS accounts for

Correspondence to: Professor Daliu Min, Department of Oncology, Shanghai Sixth People's Hospital East Campus, Shanghai Jiao Tong University, 222 Huanhu West 3 Road, Shanghai 201306, P.R. China

E-mail: scl543042177@163.com

*Contributed equally

Key words: myxoid liposarcoma, fat-bearing area, epidural region, orbit, metastasis $\sim 30 \%$ of all LPS cases and is characterized by the appearance of uniform, round- to oval-shaped cells with myxoid stroma $(1,2)$. For local disease, the 5-year disease-specific survival (DSS) rate is $93 \%$. By multivariate analysis, an age of $>45$ years, the male gender and locally recurrent disease are predictive of a poor 5-year DSS rate. For metastatic disease, the outcome is poor, with a 5-year DSS rate of $8.2 \%$. In comparison with other sarcomas, MLPS often metastasizes to the abdomen (49\%), rather than the lungs (14\%) and bones $(23 \%)$ (3). Understanding the metastasis pattern and underlying mechanism should aid in improving the survival of patients with this disease.

The current study presents a rare case of MLPS with multiple metastases in fat-bearing areas, but no involvement of the lungs. Unexpectedly, the presence of bone metastasis as diagnosed by magnetic resonance imaging (MRI) and proven by histology, was negative on bone scans. Written informed consent was obtained from the patient's family.

\section{Case report}

A 53-year-old male presented with a slowly enlarging mass in the right thigh in May 2008. The patient underwent a local excision at Shanghai General Hospital (Shanghai, China). and the histology revealed a diagnosis of MLPS. The patient subsequently received adjuvant radiotherapy (details unknown) and was free from recurrence for 34 months after the resection. The patient presented to The Sixth People's Hospital of Shanghai Jiao Tong University (Shanghai, China) on April 26, 2011, with lower back pain that radiated to the bilateral thighs for more than one month. Bone scans showed no evidence of bone metastasis. However, MRI showed abnormal signals in the vertebral body of T11 and T12, and a mass in the epidural region at the T11 and T12 vertebral level (Fig. 1). Angiography showed that the feeding vessels were the T11 and T12 intercostal arteries. Transcatheter arterial embolization was performed using polyvinylpyrrolidone and gelfoam particles, and the contrast extravasation almost disappeared in the two arteries. Next day, a laminectomy was performed and the soft tumor in the epidural region was marginally resected. Immunohistochemically, the tumor cells were positive for vimentin, slightly positive for Ki-67 (2\%), and negative for cluster of differentiation (CD) 31 , CD34, S-100, smooth muscle actin, glial fibrillary acidic 

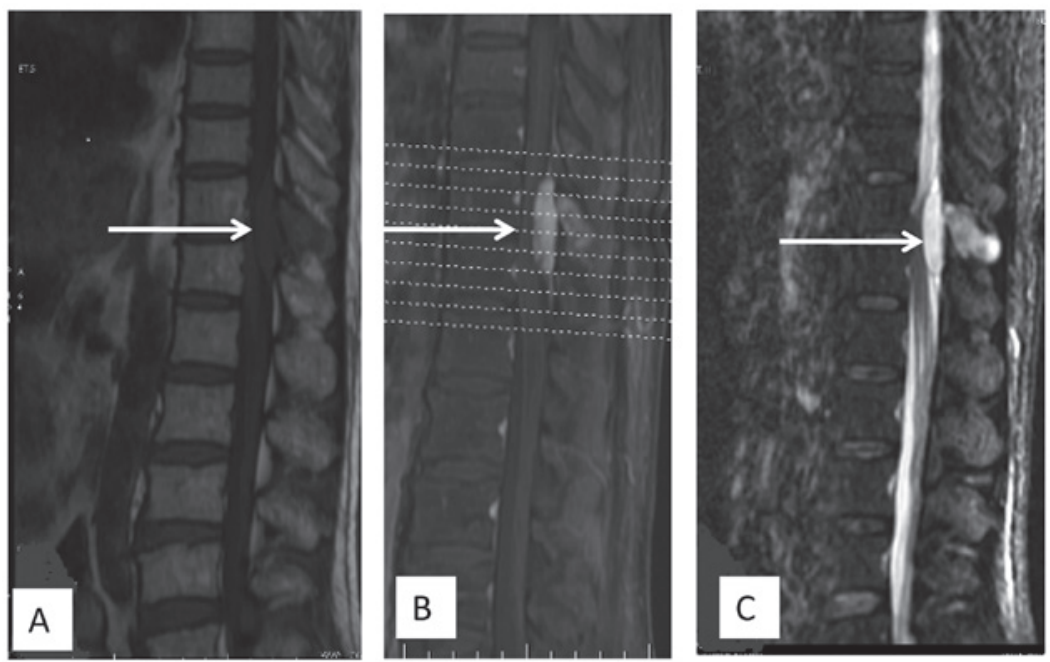

Figure 1. (A) Sagittal T1-weighted image prior to gadolinium infusion showing hypointense signals in the spinous process of the T11 vertebra and an epidural mass with hypointense signals at the T11 and T12 vertebral level. (B) Sagittal T2-weighted, fat suppressive magnetic resonance imaging revealing hyperintense signals in the spinous process of the T11 vertebra and an epidural mass with hyperintense signals at the T11 and T12 vertebral level. (C) Well-enhanced mass following gadolinium-DTPA infusion and compression of the thoracic spinal cord.
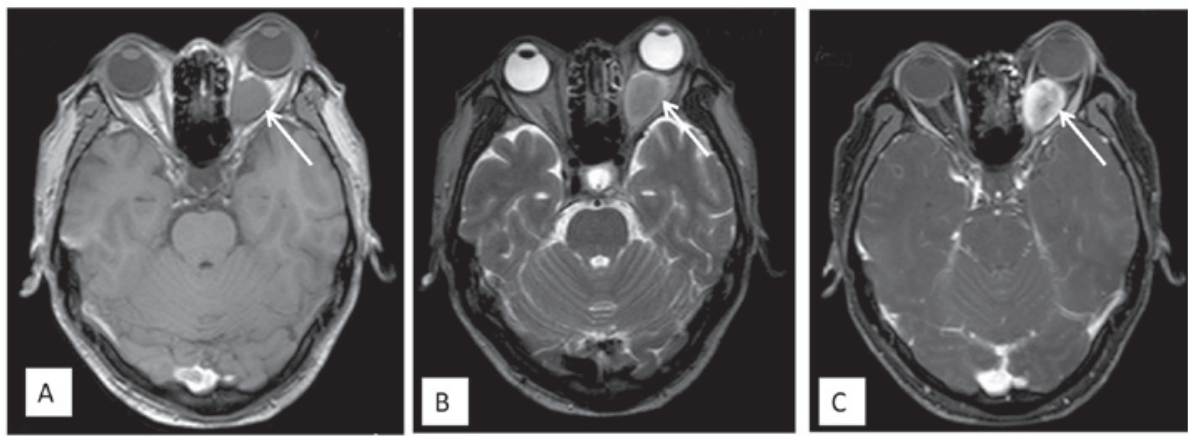

Figure 2. Axial magnetic resonance imaging showing a retrobulbar mass lesion producing (A) isointense signals on T1-weighted imaging and (B) hypo- to isointense signals on T2-weighted imaging, with (C) ring enhancement in the left eye. The mass slightly compressed the optic nerve.
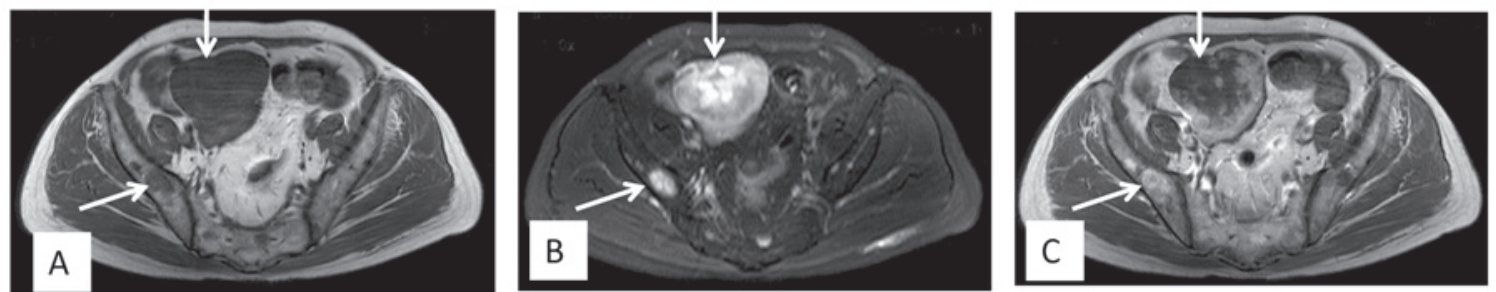

Figure 3. Axial magnetic resonance imaging showing a mass lesion producing hypointense signals on (A) T1-weighted imaging and (B) hyperintense signals on T2-weighted imaging, with (C) slight enhancement in the pelvic cavity. Also, the pelvis showed diffuse signal changes.

protein and CD56. These findings together with hematoxylin and eosin staining revealed a diagnosis of metastatic MLPS for the involved bones and the mass in the epidural region.

Following recovery from the surgery, radiotherapy was administered at 48 Gy for 24 fractions over 5 weeks. At 1 week after the initiation of radiotherapy, concurrent chemotherapy consisting of gemcitabine $\left(0.8 \mathrm{~g} / \mathrm{m}^{2}\right.$ on days 1 and 8$)$ and docetaxel $\left(75 \mathrm{mg} / \mathrm{m}^{2}\right.$ on day 8$)$ was administered. The adverse events were mild, such as leukopenia of World Health Organization grade I (4). At 16 days post-radiotherapy, a second course of chemotherapy with the same regimen was administered.
Another 18 days later, computed tomography (CT) detected a $4.5 \times 3.7-\mathrm{cm}$ mass in the abdominal cavity below the gastric body. Several days later, the patient started complaining of blurred vision and painless diplopia in the left eye. Proptosis of the left eye became progressively evident. MRI showed a retrobulbar mass, which was isointense on T1-weighted images and hypo- to isointense on T2-weighted images, with ring enhancement following gadolinium-DTPA infusion (Fig. 2). The mass slightly compressed the optic nerve. The diagnosis was of an orbital metastasis. To alleviate the eye symptoms, the patient received palliative radiotherapy 
(21 fractions of 51 Gy over 10 weeks) to the retrobulbar mass. During the radiotherapy, the patient developed multiple metastases in regions that included the pelvic cavity, the pelvis femur and the lumbar vertebrae, and progression of the orbital mass was observed (Fig. 3). However, there was no involvement of the lungs. Although the patient received second-line chemotherapy with ifosfamide $\left(1.8 \mathrm{~g} / \mathrm{m}^{2}\right.$ on days 1 to 5$)$ and epirubicin $\left(60 \mathrm{mg} / \mathrm{m}^{2}\right.$ on days 1 and 2$)$, the disease progressed and the patient's performance state deteriorated. The patient succumbed to the disease 3 months later.

\section{Discussion}

Metastasis to the orbit from LPS is rare and only four previous reports can be found in a search of the literature (5-8). The primary tumor locations were the retroperitoneum, the thigh and the abdominal cavity, and the pathological types were low/intermediate-differentiated LPS, MLPS, spindle-cell LPS or dedifferentiated LPS, respectively. Irrespective of the palliative surgical and systemic treatment, the prognosis of patients with orbit metastasis is poor.

Metastasis to the epidural region from LPS is also rare. Only three cases have been documented in the literature. The histological type of these three cases was uniformly MLPS. One case was in the cervical region and presented with liver, subcutaneous tissue metastasis (9). The second case was in the thoracic region and the patient had a history of lymph node, left buttock and left thoracic wall metastases. The patient succumbed to multiple lung metastases a few months later (10). The third case was in the thoracic region, and the patient developed abdominal metastasis and succumbed 7 weeks after the laminectomy (10). In the present case, the patient developed spinal metastases and progression with abdominal and orbital metastases 4 months after laminectomy. Collectively, these cases may suggest that epidural metastasis tends to arise from MLPS. Since the disease progressed rapidly in the patients with epidural metastasis, careful follow-up is strongly recommended to rule out metastasis in other locations.

Metastases from sarcoma are found mainly in the lungs and bones. LPS, which is the most common type of sarcoma, has an extrapulmonary metastasis pattern. The data from a study of 45 patients with MLPS suggested that the most common sites of spread were the bones, intraabdominal region, skin, mediastinum, paraspinal region and pleura (11). Cheng et al (12) also found that LPS has a tendency towards metastases occurring in the abdominal wall, axilla, breasts, subcutis, liver, dura mater, thymus and intraabdominal region. This distinct metastasis pattern was also proved by Hoffman et al (4) in a recent large-scale study of MLPS. Collectively, these results suggest that LPS, particularly MLPS, has a tendency to metastasize to fat-bearing areas (10). Concordant with this literature, the present case exhibited metastases in fat-bearing areas, including epidural, bone, abdominal and orbital metastases. However, the reason for this tendency is not clear. Hoffman et al (4) completed a pilot study concerning the molecular characteristics of MLPS. The study showed that MLPS expressed high levels of adipophilin, peroxisome proliferator-activated receptor- $\gamma$ (PPAR- $\gamma$ ), chemokine (C-X-C motif) receptor 4 (CXCR4), AXL receptor tyrosine kinase and platelet-derived growth factor receptor- $\beta$
(PDGFR- $\beta$ ). The aforementioned molecules were correlated with adipogenesis, migration, invasion, angiogenesis and metastasis. Further investigation is warranted to determine whether adipophilin and CXCR4 molecules contributed to the unique metastatic pattern.

In the present case, spinal metastasis was detected by MRI, although bone scans were negative. Bone scans normally detect skeletal metastasis using technetium-99m methylene diphosphonate binding to osteoblastic cells. For the majority of other cancer types, it is a routine procedure for patients during follow-up screening, as it is more sensitive than plain radiographs and $\mathrm{CT}$ for finding skeletal metastasis. However, for MLPS, several previous studies reported that skeletal metastasis was detectable by MRI, but not by bone scans (13-17). In one previous study, based on whole-body MRI findings, 33 MLPS patients were identified with spinal metastases. These metastases were detected by bone scans in $16 \%$ of patients and by positron emission tomography scans in $14 \%$ of patients (1). The reason why bone scanning is not a reliable method to detect skeletal metastasis remains unclear. We hypothesize one possible reason for this may be that there are few osteoblastic cells in skeletal metastasis. Recently, a variant chromosomal translocation $\mathrm{t}(12 ; 22)(\mathrm{q} 13 ; \mathrm{q} 12)$ was identified. This translocation results in the production of the Ewing sarcoma breakpoint region 1-DNA damage-inducible transcript 3 (EWSR1-DDIT3) fusion protein. This fusion protein exerted a selective effect on the transcriptional activity of cell lineage-specific marker genes in multipotent mesenchymal C3H10T1/2 cells. The osteoblastic marker Opn promoter was repressed, while the adipocytic marker PPAR- $\gamma 2$ promoter was not affected. The study concluded that EWSR1-DDIT3 may contribute to the phenotypic selection of mesenchymal cells during MLPS initiation and development (18). We propose that by the effect of this protein, mesenchymal cells in skeletal metastasis mainly transformed to adipocyte-related cells, but few osteoblastic cells. Therefore, this histological change may result in ineffective detection of the skeletal metastasis by bone scans.

In summary, a case of MLPS with skeletal, epidural, orbital and abdominal cavity metastases was reported. MRI clearly showed an abnormal signal in the vertebral body, however, bone scans were negative. Recent results have suggested that MLPS expresses high levels of adipophilin, PPAR- $\gamma$, CXCR4, AXL receptor tyrosine kinase, PDGFR- $\beta$ and may present with the EWSR1-DDIT3 fusion transcript. We hypothesize that these genetic and molecular changes may contribute to the distinct biological characteristics of MLPS.

\section{References}

1. Schwab JH, Boland PJ, Antonescu C, Bilsky MH and Healey JH: Spinal metastases from myxoid liposarcoma warrant screening with magnetic resonance imaging. Cancer 110: 1815-1822, 2007.

2. Sanfilippo R, Dei Tos AP and Casali PG: Myxoid liposarcoma and the mammalian target of rapamycin pathway. Curr Opin Oncol 25: 379-383, 2013.

3. Hoffman A, Ghadimi MP, Demicco EG, Creighton CJ, Torres K, Colombo C, Peng T, Lusby K, Ingram D, Hornick JL, et al: Localized and metastatic myxoid/round cell liposarcoma: Clinical and molecular observations. Cancer 119: 1868-1877, 2013.

4. .Miller AB, Hoogstraten B, Staquet M and Winkler A: Reporting results of cancer treatment. Cancer 47: 207-214, 1981 
5. Fabi A, Salesi N, Vidiri A, Mirri A, Ferraresi V and Cognetti F: Retroperitoneal liposarcoma with metastasis to both orbits: An unusual metastatic site. Anticancer Res 25: 4769-4771, 2005.

6. Abdalla MI, Ghaly AF and Hosni F: Liposarcoma with orbital metastases. Case report. Br J Ophthalmol 50: 426-428, 1966.

7. Tehrani AH, Heegaard S, Prause JU, Fledelius HC and Daugaard S: Liposarcoma metastatic to the orbit. Eur J Ophthalmol 13: 108-112, 2003.

8. Fezza J and Sinard J: Metastatic liposarcoma to the orbit. Am J Ophthalmol 123: 271-272, 1997.

9. Lee SY, Kim HJ, Park SY, Park YH and Chung SK: Myxoid liposarcoma involving the liver, subcutaneous tissue and epidural space in a polycystic disease patient. Clin Nucl Med 33: 507-509, 2008.

10. Ogose A, Hotta T, Inoue Y, Sakata S, Takano R and Yamamura S Myxoid liposarcoma metastatic to the thoracic epidural space without bone involvement: Report of two cases. Jpn J Clin Oncol 31: 447-449, 2001.

11. Fuglø HM, Maretty-Nielsen K, Hovgaard D, Keller JØ, Safwat AA and Petersen MM: Metastatic pattern, local relapse and survival of patients with myxoid liposarcoma: A retrospective study of 45 patients. Sarcoma 2013: 548628, 2013

12. Cheng EY, Springfield DS and Mankin HJ: Frequent incidence of extrapulmonary sites of initial metastasis in patients with liposarcoma. Cancer 75: 1120-1127, 1995.
13. Sakamoto A, Fukutoku Y, Matsumoto Y, Harimaya K, Oda Y and Iwamoto Y: Myxoid liposarcoma with negative features on bone scan and 18F-2-fluoro-2-deoxy-D-glucose-positron emission tomography. World J Surg Oncol 10: 214, 2012.

14. Conill C, Setoain X, Colomo L, Palacín A, Combalia-Aleu A, Pomés J, Marruecos J, Vargas M and Maurel J: Diagnostic efficacy of bone scintigraphy, magnetic resonance imaging and positron emission tomography in bone metastases of myxoid liposarcoma. J Magn Reson Imaging 27: 625-628, 2008.

15. Khurana JS, Rosenthal DI, Rosenberg AE and Mankin HJ Skeletal metastases in liposarcoma detectable only by magnetic resonance imaging. Clin Orthop Relat Res 204-207, 1989.

16. Ishii T, Ueda T, Myoui A, Tamai N, Hosono N and Yoshikawa $\mathrm{H}$ : Unusual skeletal metastases from myxoid liposarcoma only detectable by MR imaging. Eur Radiol 4: L185-L191, 2003.

17. Kato S, Kawahara N, Murakami H, Demura S, Shirai T, Tsuchiya $\mathrm{H}$ and Tomita K: Multi-level total en bloc spondylectomy for solitary lumbar metastasis of myxoid liposarcoma. Orthopedics 33: 446, 2010.

18. Suzuki K, Matsui Y, Higashimoto M, Kawaguchi Y, Seki S, Motomura H, Hori T, Yahara Y, Kanamori M and Kimura T: Myxoid liposarcoma-associated EWSR1-DDIT3 selectively represses osteoblastic and chondrocytic transcription in multipotent mesenchymal cells. PLoS One 7: e36682, 2012. 